\title{
Erratum to: Quality assessment of digested sludges produced by advanced stabilization processes
}

\author{
C. M. Braguglia • A. Coors • A. Gallipoli • A. Gianico • \\ E. Guillon • U. Kunkel • G. Mascolo • E. Richter • \\ T. A. Ternes • M. C. Tomei • G. Mininni
}

Published online: 7 February 2015

(C) Springer-Verlag Berlin Heidelberg 2015

Erratum to: Environ Sci Pollut Res

DOI 10.1007/s11356-014-3090-6

The $2^{\text {nd }}$ affiliation of Elisabeth Richter is missing.

She is also affiliated to: Goethe-University Frankfurt/Main, Department Aquatic Ecotoxicology, Frankfurt/Main, Germany

The online version of the original article can be found at http://dx.doi.org/ 10.1007/s11356-014-3090-6.

C. M. Braguglia $(\bowtie) \cdot$ A. Gallipoli · A. Gianico $\cdot$ M. C. Tomei

G. Mininni

Istituto di Ricerca sulle Acque-CNR, Area della Ricerca RM1,

Monterotondo, Rome, Italy

e-mail: braguglia@irsa.cnr.it

A. Coors $\cdot$ E. Richter

ECT Oekotoxikologie, Flörsheim/Main, Germany

E. Guillon

ICMR UMR CNRS 7312, Université de Reims

Champagne-Ardenne, Reims, France

U. Kunkel · T. A. Ternes

Federal Institute of Hydrology (BfG), Koblenz, Germany

G. Mascolo

Istituto di Ricerca sulle Acque-CNR, Bari, Italy

E. Richter

Goethe-University Frankfurt/Main, Department Aquatic

Ecotoxicology, Frankfurt/Main, Germany 